\title{
Multi-atlas active contour segmentation method using template optimization algorithm
}

\author{
Monan Wang*, Pengcheng Li and Fengjie Liu
}

\begin{abstract}
Background: Brain image segmentation is the basis and key to brain disease diagnosis, treatment planning and tissue 3D reconstruction. The accuracy of segmentation directly affects the therapeutic effect. Manual segmentation of these images is time-consuming and subjective. Therefore, it is important to research semi-automatic and automatic image segmentation methods. In this paper, we propose a semi-automatic image segmentation method combined with a multi-atlas registration method and an active contour model (ACM).

Method: We propose a multi-atlas active contour segmentation method using a template optimization algorithm. First, a multi-atlas registration method is used to obtain the prior shape information of the target tissue, and then a label fusion algorithm is used to generate the initial template. Second, a template optimization algorithm is used to reduce the multi-atlas registration errors and generate the initial active contour (IAC). Finally, a ACM is used to segment the target tissue.

Results: The proposed method was applied to the challenging publicly available MR datasets IBSR and MRBrainS13. In the MRBrainS13 datasets, we obtained an average thalamus Dice similarity coefficient of $0.927 \pm 0.014$ and an average Hausdorff distance (HD) of $2.92 \pm 0.53$. In the IBSR datasets, we obtained a white matter (WM) average Dice similarity coefficient of $0.827 \pm 0.04$ and a gray gray matter (GM) average Dice similarity coefficient of $0.853 \pm 0.03$.

Conclusion: In this paper, we propose a semi-automatic brain image segmentation method. The main contributions of this paper are as follows: 1) Our method uses a multi-atlas registration method based on affine transformation, which effectively reduces the multi-atlas registration time compared to the complex nonlinear registration method. The average registration time of each target image in the IBSR datasets is $255 \mathrm{~s}$, and the average registration time of each target image in the MRBrainS13 datasets is $409 \mathrm{~s}$. 2) We used a template optimization algorithm to improve registration error and generate a continuous IAC. 3) Finally, we used a ACM to segment the target tissue and obtain a smooth continuous target contour.
\end{abstract}

Keywords: Multi-atlas segmentation, Images registration, Active contour model, Template optimization

\section{Background}

Brain image segmentation is crucial for the diagnosis and treatment of brain diseases, and it is the basis of the three-dimensional reconstruction of brain structure and quantitative analysis of lesions. The accuracy of segmentation directly affects focus tissue localization, lesion

\footnotetext{
* Correspondence: mnwang@hrbust.edu.cn

School of Mechanical \& Power Engineering, Harbin University of Science and Technology, Xue Fu Road No. 52, Nangang District, Harbin City, Heilongjiang Province 150080, People's Republic of China
}

shape and size measurement, and clinical diagnosis and treatment planning.

Many brain diseases are manifested as changes in the normal volume and regional distribution of the WM, GM and cerebrospinal fluid (CSF), such as Alzheimer's disease [1], which is characterized by atrophy of the whole brain, decrease in volume, narrowing of the brain at the cortex, and deepening of the cerebral sulci. Multiple sclerosis [2] shows ventricular enlargement and WM encroaching on GM. The thalamus is associated with epilepsy, and deep brain stimulation of thalamic nuclei is being developed as

(C) The Author(s). 2019 Open Access This article is distributed under the terms of the Creative Commons Attribution 4.0 International License (http://creativecommons.org/licenses/by/4.0/), which permits unrestricted use, distribution, and 
a treatment for drug- resistant epilepsy [3]. Reliably identifying the thalamus can increase the success rate of treatment. The thalamus belongs to the GM and is connected to not only the WM and CSF but also other structures belonging to the GM. This causes the boundaries of the thalamus to be inconspicuous and difficult to identify. Therefore, accurately identifying and analysing changes in the volume, shape, size, and grayscale distribution of these brain structures is very important for clinical diagnosis and treatment.

The image segmentation method based on the ACM [4] has a simple expression and high computational efficiency, and by this method, a smooth continuous target contour can be obtained. In recent decades, ACM has been successfully applied to image edge detection, image segmentation and motion tracking [5]. Chakraborty [6] integrated gradient and region information to achieve the target contour segmentation and showed that the integrated method performs better than conventional gradient-based image segmentation. However, ACM is very sensitive to the initial contour, and it is difficult to achieve accurate segmentation for target tissues with large intensity inhomogeneities and complex shapes. An effective way to solve this problem is to use prior knowledge of shape information to constrain contour deformation [7].

In recent years, multi-atlas segmentation (MAS) methods have become the basic tool for image segmentation. MAS methods can transfer the prior information of the atlas to the target image, and the segmentation accuracy is equivalent to that of manual segmentation $[8,9]$. MAS methods use multi-atlas registration to select reference labels for the target image. The reference labels are manually created by the doctor according to the atlas images. Then, the transformation parameters obtained by multi-atlas registration are used to warp the reference labels. Finally, a label fusion method is used to correct the errors of each label. In recent years, many algorithms based on the MAS framework have been proposed, with particular emphasis on label fusion and atlas selection [10-12].

Artaechevarria [13] proposed a generalized local weighting voting (WV) method, which improved the segmentation accuracy in segmenting high-contrast structures compared to the global WV method. However, the contour obtained by the multi-atlas-based segmentation method is not smooth and is prone to breakpoints. Thomas et al. [14] proposed a selective and iterative method for performance level estimation (SIMPLE), which combines atlas selection and performance estimation strategies to achieve the segmentation of the target organization. Jimit et al. [15] proposed a multi-atlas region segmentation utilizing ensembles (MUSE), which improves segmentation accuracy by linear combinations of different atlas selections, deformation algorithms and deformation parameters. Zhang et al. [16] proposed a multi-atlas level set framework (MALSF) segmentation by combining the ACM and MAS method. This method uses multi-atlas registration to obtain the a priori information of the target tissue and then uses the level set algorithm to segment the target tissue and obtain a smooth, continuous contour.

Learning-based image segmentation methods have been shown to achieve satisfactory results when applied to image segmentation problems. Bai et al. [17] proposed a segmentation method combining augmented features and support vector machines (SVMs) and improved the performance of non-local patch-based segmentation. Zikic and Glocker [18] proposed a multi-atlas label propagation method based on randomized classification forests. Convolutional neural networks (CNNs) have shown superiority in several computer vision tasks, such as the ImageNet challenge [19]. Recently, CNNs have also been prevalent in medical image analysis [20] due to their flexibility. Moeskops et al. [21] presented a multi-scale patch-wise CNNs approach to segment brain images. It simplified tissue segmentation into a classification problem by extracting the patch in voxels and judging which tissue it belonged to. Subsequently, Nie et al. [22] employed fully convolutional networks (FCNs) to segment the isointense phase brain MR images. Hou et al. [23] proposed a 3D Convolutional Neural Network Segmentation Method Combined with Boundary Correction $(\mathrm{BC}-\mathrm{CNN})$. They used densely $\mathrm{CNNs}$ to roughly classify the imaged tissue and then detailed the boundaries generated in the previous stage.

In this paper, we propose a multi-atlas active contour segmentation method using a template optimization algorithm. The proposed method combines the advantages of a multi-atlas and ACM and can effectively utilize prior knowledge of the atlas and obtain a smooth target tissue contour. It is closer to reality and facilitates the three- dimensional reconstruction of the target tissue; thus, it is convenient for the doctor to observe the volume and morphological changes of the target tissue. To verify the segmentation performance of our method, we tested our method on the IBSR and MRBrainS13 datasets and employed four commonly used metrics, Dice, Recall, Precision and HD, to evaluate the similarity between segmentation results and manual labels. The results show that our method obtains satisfactory segmentation accuracy.

\section{Method}

The overall framework of our method is summarized in Fig. 1. Our method has four steps: multi-atlas registration, label fusion, template optimization and ACM. Each step of our method is described in detail in the following subsections. 


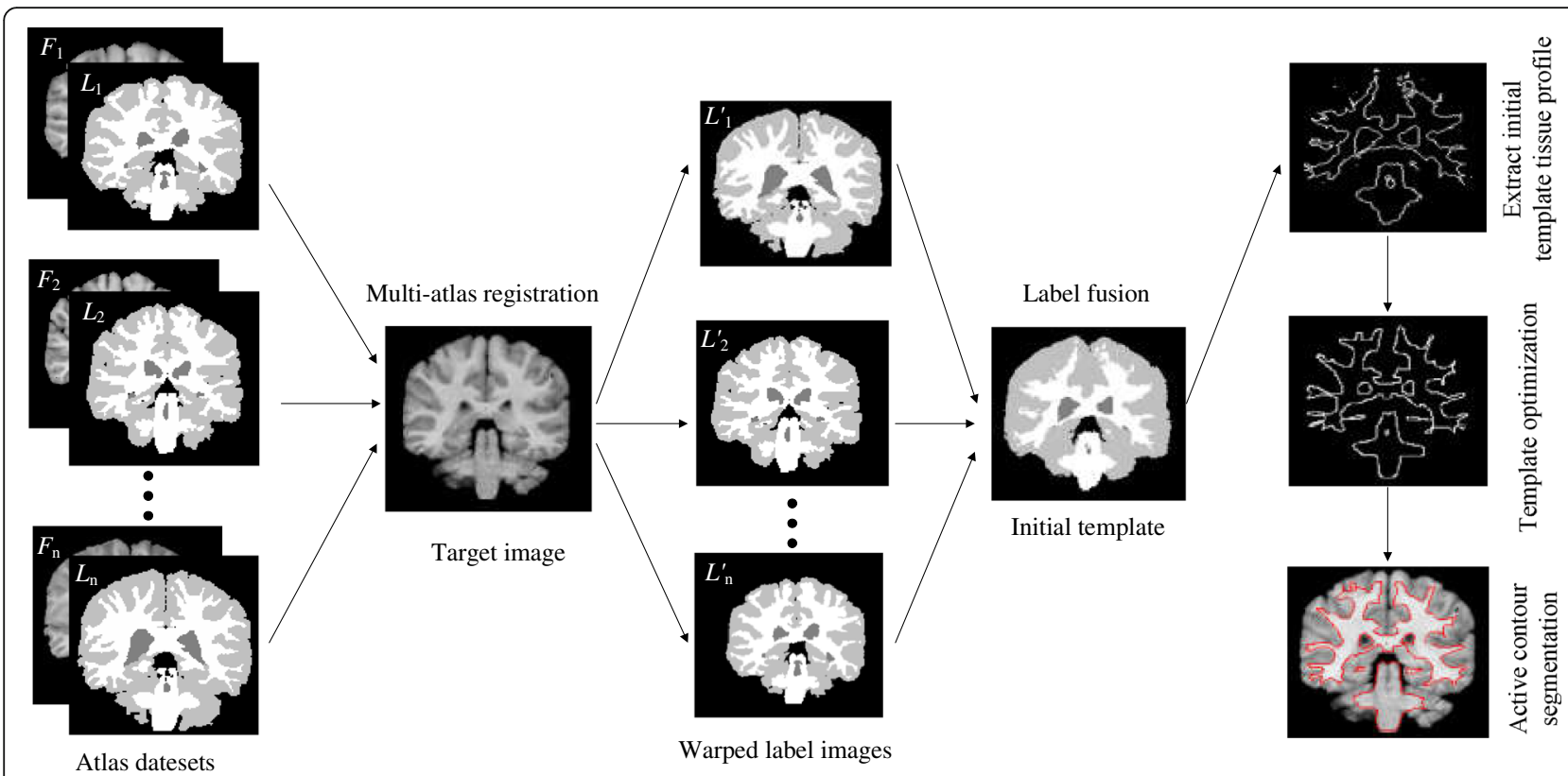

Fig. 1 Schematic diagram of multi-atlas active contour segmentation method using template optimization algorithm

Multi-atlas registration

An atlas contains an intensity image and a reference label that is manually divided by the doctors. In multi-atlas image registration, the intensity images are registered with the target image to obtain transformation parameters; then, the transformation parameters are used to warp the label images to spatially correspond to the target image. Finally, the warped label images are fused to generate the initial template. The multi-atlas-based segmentation method can effectively reduce the influence of individual atlas registration errors on segmentation results because a single atlas registration error may be rejected during label fusion.

In this paper, we use a global registration method based on affine transformation and then use a label fusion and template optimization algorithm to correct the errors caused by registration. The affine transform is defined as follows:

$$
f^{\prime}(x, y)=K\left[\begin{array}{cc}
\cos \theta & -\sin \theta \\
\sin \theta & \cos \theta
\end{array}\right] f(x, y)+\Delta X
$$

Where $f(x, y)$ is pixel coordinate, $\mathrm{K}$ is scaling parameter, $\theta$ is rotation angle and $\Delta x$ is translation parameters. Considering the IBSR datasets from different magnetic imaging devices, there is non-standard intensity between images, therefore, we use normalized correlation coefficient (NCC) as the similarity measure function:

$$
\begin{gathered}
N C C(\tau, T I, F I)=\frac{\sum_{i=1}^{n}\left(T I\left(x_{i}\right)-\overline{T I}\right) \times \sum_{i=1}^{n}\left(F I\left(\tau\left(x_{i}\right)\right)-\overline{F I(\tau)}\right)}{\sqrt{\sum_{i=1}^{n}\left(T I\left(x_{i}\right)-\overline{T I}\right)^{2} \times \sum_{i=1}^{n}\left(F I\left(\tau\left(x_{i}\right)\right)-\overline{F I(\tau)}\right)^{2}}} \\
\overline{T I}=\sum_{i=1}^{n} T I\left(x_{i}\right) / n \\
\overline{F I(\tau)}=\sum_{i=1}^{n} F I\left(\tau\left(x_{i}\right)\right) / n
\end{gathered}
$$

Where $\operatorname{TI}\left(x_{i}\right)$ is the gray value at pixel point $x_{i}$ in the target image, $F I\left(x_{i}\right)$ is the gray value at pixel point $x_{i}$ in the warped floating image and $n$ represents the number of image pixels. The NCC assumes that there is a certain linear relationship between the two images, and it is widely used in single-modality medical image registration.

\section{Label fusion}

After image registration, the target image pixels must be assigned a label. In multi-atlas registration, many atlas labels are generated; therefore, a label fusion algorithm is needed to fuse a plurality of warped label images to obtain a clear and accurate fusion image.

Label fusion is a key step in a multi-atlas-based registration segmentation method, and it can discard pixels with low agreement between different propagation labels to minimize outliers. Therefore, label fusion can effectively improve segmentation accuracy, but it may lead to slight distortions between the images.

In recent years, research on segmentation based on a multi-atlas has mainly focused on the label fusion method. 
Some label fusion methods have been proposed, such as majority voting [11], local WV [13], and probabilistic atlases [24], etc.

Voting rules are typically applied because they are computationally simple. Considering that there are large local differences in different atlas images, in this paper, we use a local WV algorithm to assign the appropriate label to the target image.

$$
S(x)=\arg \max _{c} \sum_{i=1}^{L} w_{i}(x) \cdot f\left(M_{i}^{\prime}(x), c\right)
$$

Where c represents a class, $L$ is the number of atlases, $w_{i}(x)$ is a weight function at pixel $\mathrm{x}$, and each pixel is given a different weight according to the NCC similarity coefficient of the local area between atlas image and target image, in this paper, the local area size is set to $3 \times 3$. The $f\left(M_{i}^{\prime}(x), c\right)$ is defined as:

$$
f\left(M_{i}^{\prime}(x), c\right)=\left\{\begin{array}{cc}
1 & M_{i}^{\prime}(x)=c \\
0 & M_{i}^{\prime}(x) \neq c
\end{array}\right.
$$

\section{Template optimization}

The generated initial template is generally similar to the target label image; however, there is still a large local error. In our method, we use a template optimization algorithm to correct registration errors. It mainly consists of three steps: search area setting, contour point determination, and IAC acquisition.

\section{Search area setting}

There are three steps in the search area setting:

1. Extracting the WM contour of the initial template

2. Selecting a certain size rectangular area as the initial search area centred on each pixel on the contour

3. Determining whether each pixel in the search area belongs to the contour boundary according to the grayscale and gradient information of the target image

To avoid search area overlap problems from two adjacent outlines, we adjust the scope of the search area. As shown in Fig. 2, if there is an outline that is not adjacent to the central pixel in the $x$-direction or $y$-direction in the initial searched area, the search area boundary value is set to the middle value between the two contour lines. The adjusted search area is then defined as the actual search area.

\section{Contour point determination}

To avoid the effect of noise, we use a Gaussian kernel function with a standard deviation of 0.5 to smooth the target image and the grayscale images in the IBSR datasets. We

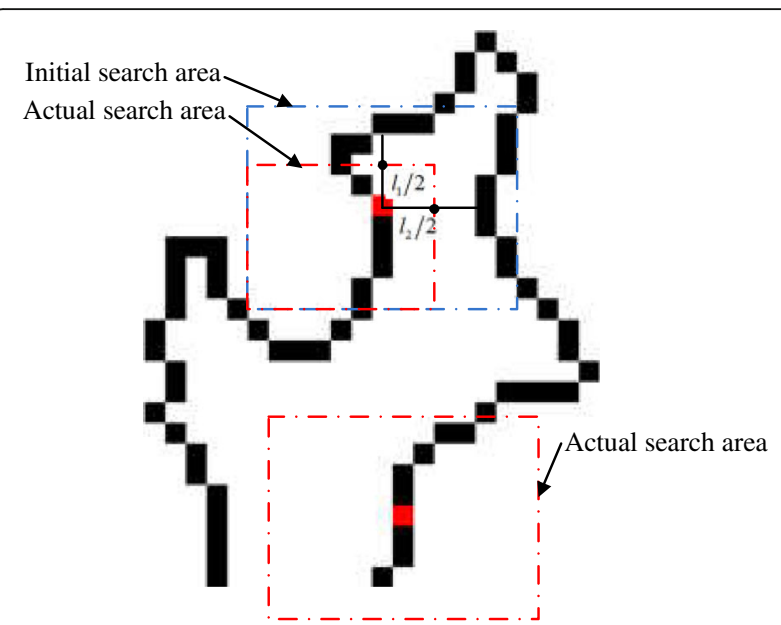

Fig. 2 Search area setting: black curve is tissue outline, red pixel is center pixel, blue dotted line is initial search area, and the red dotted line is the actual search area

also perform pixel value normalization on these images. Because the boundary gradient between WM and GM is small, it is not ideal to determine whether a pixel is a contour point based on the local gradient value. Therefore, we introduce the gray information of the target image. We extract the intensity distribution map of the pixels on the WM contour of the IBSR datasets. As shown in Fig. 3, the intensity distribution map approximates a normal distribution. The pixel value from 0 to 1 is evenly divided into 20 intervals, and an intensity weight is assigned for each pixel according to the intensity distribution map. The weight value is defined as follows:

$$
\begin{aligned}
& P(x)=\frac{p r(x)-\min p r}{\max p r-\min p r} \\
& \operatorname{pr}(x)=n_{i} / \sum_{i=1}^{N} n_{i}
\end{aligned}
$$$$
i=1,2 \cdots 20
$$

Where $i$ is the pixel value interval number corresponding to the pixel $x, n_{i}$ is the number of pixel points in the interval $i$.

Because the MR image's intensity is not constant for each tissue type, this results in a large gradient change at the WM profile. It is difficult to find a suitable gradient threshold to identify the contour boundary; to address this problem, we use the Robert edge detection operator [25] to calculate the gradient of each pixel in the actual search area and normalize the gradient. Then, we combine the intensity weight and gradient value to determine if the pixel is a contour point. The decision formula is defined as follows: 


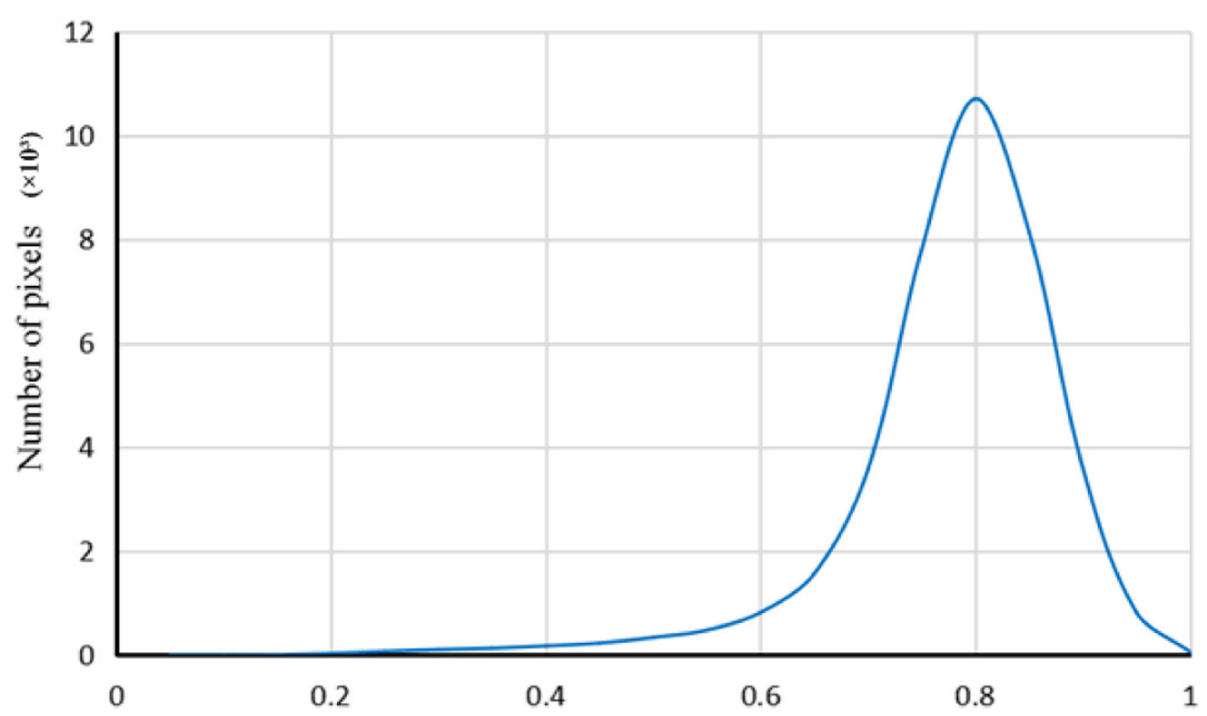

Fig. 3 Intensity distribution map of the pixels on the WM contour of the IBSR datasets

$$
Y=w_{p} \cdot P(x)+w_{g} \cdot \frac{\operatorname{grad}(x)-\min \operatorname{grad}}{\max \operatorname{grad}-\min \operatorname{grad}}
$$

where $\operatorname{grad}()$ is the pixel gradient and are weight coefficients. The range of $\mathrm{Y}$ is from 0 to 1 . The larger the $\mathrm{Y}$ value, the greater the probability that the pixel point is the contour point. The tissue contour within the actual search area can be extracted by setting a suitable threshold for Y.

\section{IAC acquisition}

In this paper, we use a parameter ACM to segment the target tissue. The parameter ACM needs to set a continuous IAC curve. However, the contour points obtained by the above method are not continuous, and there is no topological relationship between each point. To solve this problem, we use the contour points obtained in the above method to generate a tissue contour image and then add the topological relationship for each point according to the tissue contour image to obtain the IAC.

\section{Active contour model}

In order to obtain smooth and accurate segmentation results and effectively use the prior information of the atlas images and the gray information of the target image, we use the parametric ACM to further correct the errors caused by the multi-atlas registration process. The energy equation is as follows:

$$
E_{\text {snake }}(v(s))=\int_{0}^{1}\left(E_{\text {int }}(v(s))+E_{\text {ext }}(v(s))\right) d s
$$

The ACM is actually a functional of a plane curve, and the target contour curve is the local minimum of the functional. Where $E_{i n t}$ is the internal energy term and is defined as follows.

$$
E_{\text {int }}(v(s))=\frac{1}{2} \int \alpha(s)\left|v^{\prime}(s)\right|^{2}+\beta(s)\left|v^{\prime \prime}(s)^{2}\right| d s
$$

Where $v^{\prime}(s)$ is the first order derivative of the IAC, it represents the elastic energy of the curve. The $v^{\prime \prime}(s)$ is the second order derivative of the IAC, it represents the bending energy of the curve. $\alpha(s), \beta(s)$ are weight parameters, $\alpha(s)$ controls the continuity of the IAC and $\beta(s)$ controls the smoothness of the IAC.

$E_{\text {ext }}$ is the external energy term, and it is used to pull the IAC toward target image edges. The definition of the $E_{\text {ext }}$ is the key to the ACM. In our method, the $E_{\text {ext }}$ is defined as follows:

$$
E_{\text {ext }}(v(s))=\int_{\Omega} \gamma(s) E_{i m g}(v(s)) d s
$$

where $E_{i m g}$ is a scalar potential function defined on the image plane [26], $\gamma(s)$ is weight parameter of $E_{i m g}$. Generally, the gradient of the target image is used to build the $E_{i m g}$. But there is a restriction that the $E_{i m g}$ sometimes will converge the target contour to the wrong border, such as the border between the background and the brain tissue. To avoid this problem, we combine image grayscale and gradient information to build the $E_{i m g}$ : 


$$
E_{\text {img }}(x)=\left|\nabla\left(G_{\sigma} * I(x)\right)^{2}+P(x)\right|
$$

where $I(x)$ is the initial grayscale image, $G_{\sigma}$ is a Gaussian smoothing filter. The $\nabla$ symbol represents the gradient operator.

\section{Results}

In this section, we tested our method on the groundtruthed IBSR datasets and MRBrainS13 datasets. The image datasets and the performance of each step of our method are described in detail in the following subsections.

\section{Data and segmentation evaluation index}

We tested our method using multi-center image data with a total of 55 image datasets from multiple scanners in the IBSR datasets [27] and MRBrainS13 datasets. Multi-center image data are superior to a single center in that the former contain a large amount of image data from different regions, different people, and different scanners. These factors will make the image intensity and structure different. Therefore, the application of multi-center image data can better evaluate the applicability and segmentation performance of different segmentation methods.

The IBSR datasets contain 38 normal T1-weighted MR brain datasets and their reference regions of interest (ROI) labels, which are defined by the Center for Morphometric Analysis at Massachusetts General Hospital, and all the data were publicly available. Twenty datasets are low-resolution data whose dimensions are $256 \times$ $256 \times 60$, and the remaining eighteen datasets are high-resolution data whose dimensions are $256 \times 256 \times$ 128. In the low-resolution datasets, organizational boundaries are more difficult to identify. In our method, a template optimization algorithm is used to identify this boundary information. To verify the validity of our method, we tested it in the 20 low-resolution datasets.

MRBrainS13 datasets were provided by https://masi. vuse.vanderbilt.edu/. This website provides the online continuation of a segmentation contest held at the 2013 Medical Image Computing and Computer Assisted Intervention Challenge (MICCAI). There are 35 T1-weighted MR brain image datasets and their label images. The dimensions of all datasets are $256 \times 256 \times 287$.

We employed three commonly used metrics, Dice, Recall and Precision, to measure the volumetric overlap of the segmentation results with manual labels, and we used HD to measure the surface distance between segmentation results and manual labels. These metrics are defined as follows:

$$
\begin{aligned}
\operatorname{Dice}(T, F) & =\frac{2 V(T \cap F)}{V(T)+V(F)} \quad \operatorname{Recall}(T, F)=\frac{V(T \cap F)}{V(T)} \\
\text { ecision }(T, F) & =\frac{V(T, F)}{V(F)}(14) \\
H D(T, F) & =\max \left(H_{1}(T, F), H_{2}(F, T)\right)
\end{aligned}
$$

$$
\begin{aligned}
& H_{1}(T, F)=\max _{p_{t} \in T}\left(\min _{p_{f} \in F} d\left(p_{t}, p_{f}\right)\right) \\
& H_{2}(F, T)=\max _{p_{f} \in F}\left(\min _{p_{t} \in T} d\left(p_{f}, p_{t}\right)\right)
\end{aligned}
$$

Where $T$ is the target tissue pixel set in the target label image, and $F$ is the segmented target tissue pixel set. $H D(T, F)$ is the HD between the pixel set $T$ and $F, d\left(p_{t}, p_{f}\right)$ is the distance between pixel $p_{t}$ and $p_{f}$.

We used a leave-one-out cross-validation method to test the performance of our method on the IBSR and MRBrainS13 datasets. One MR brain dataset was selected as the target set, and the remaining MR brain datasets were treated as training sets; this was repeated until each MR brain dataset was used as a target set.

\section{Label fusion}

To reduce the influence of background pixels on the registration result, we took the smallest rectangular area that contains all the target image brain tissues as the ROI. In this step, we selected the label images with NCC values greater than 0.87 after image registration as the label images corresponding to the target image. We then used local WV to fuse these label images to generate the initial template with the binarization threshold set to 0.5 .

Figure 4 shows the results obtained during label fusion and template optimization. It can be seen from (c) and (b) that the generated initial template is generally similar to the target label image, but some small WM tissues in the brain returned are lost in the IBSR datasets, and some GM tissues are misdivided into the thalamus in the MRBrainS13 datasets. To reduce these errors, we used a template optimization algorithm.

\section{Template optimization}

In this step, we used the template optimization algorithm to optimize the initial template. The optimization parameters are shown in Table 1. In Fig. 4, the (d), (e), and (f) images show the changes in the target tissue contour during the template optimization process. (f) is the template-optimized IAC; compared to the contour in (e), (f) adds some small target tissue contours and removes some of the wrong incorrect contour points. The IAC is a smooth continuous contour for the initial contour setting of the next step. Figure 5 shows the Dice similarity coefficients of the initial template and the optimized template. After template optimization, the Dice similarity coefficients were significantly improved.

\section{Experimental results}

A parameter ACM was used to drive the IAC deformation to segment the target tissue, and the parameter settings are shown in Table 1 . Table 2 shows the performance of 

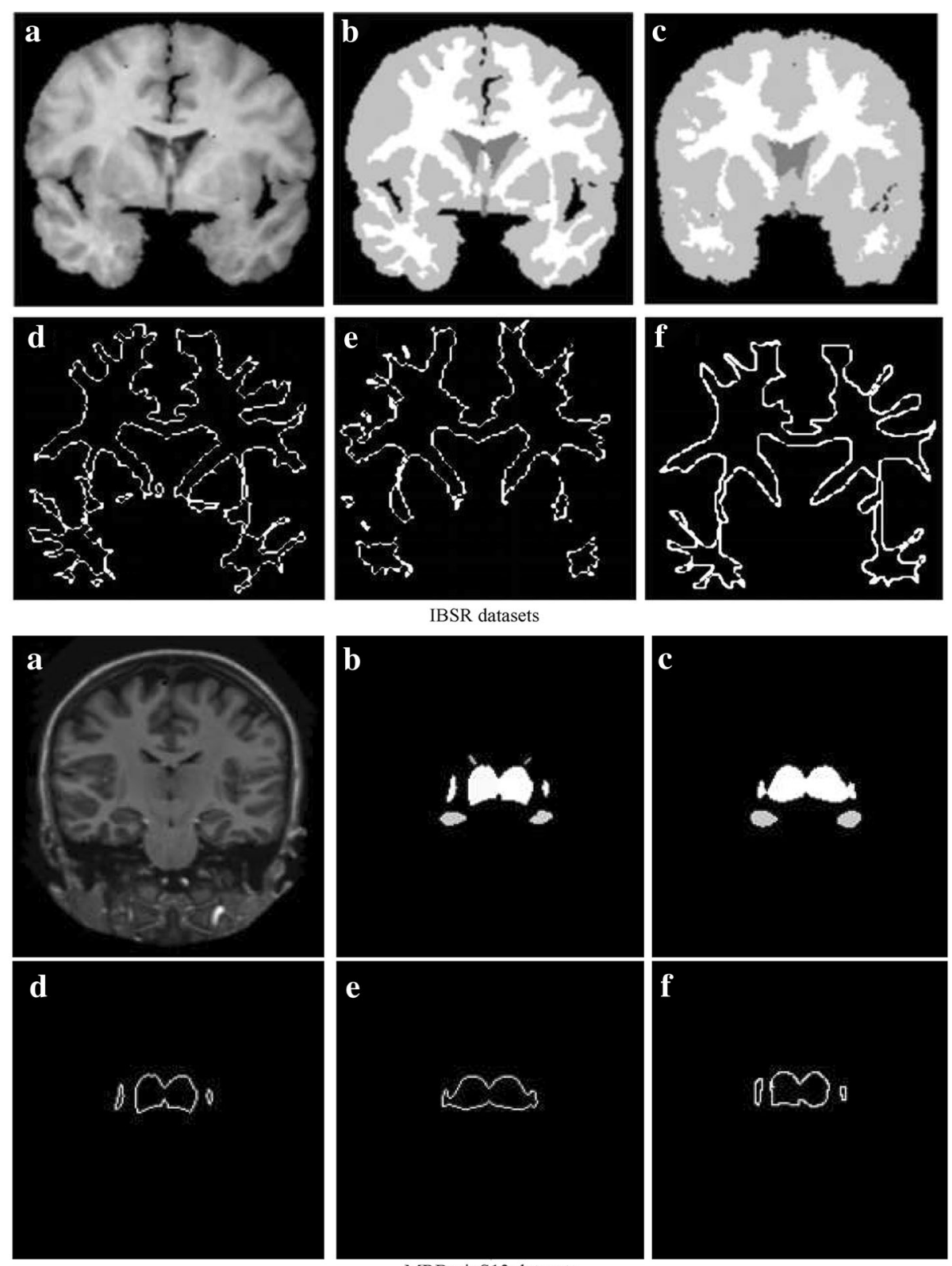

MRBrainS13 datasets

Fig. 4 Label fusion and Template optimization: a target image. b Target label image. $\mathbf{c}$ initial template. $\mathbf{d}$ Target tissue contour in label image. e Target tissue contour in initial template. $\mathbf{f}$ generated IAC

Table 1 The parameters used in all experiments

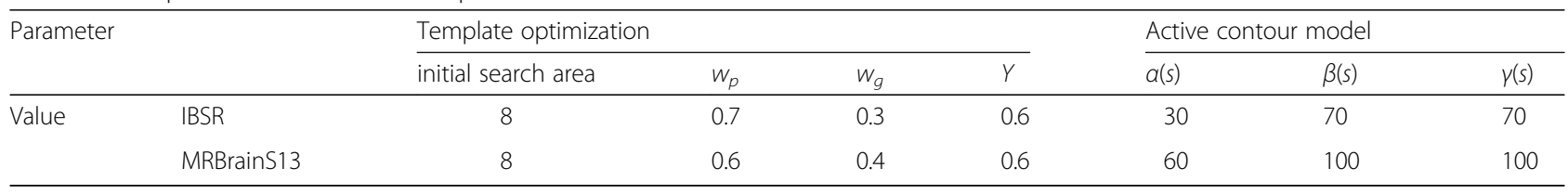




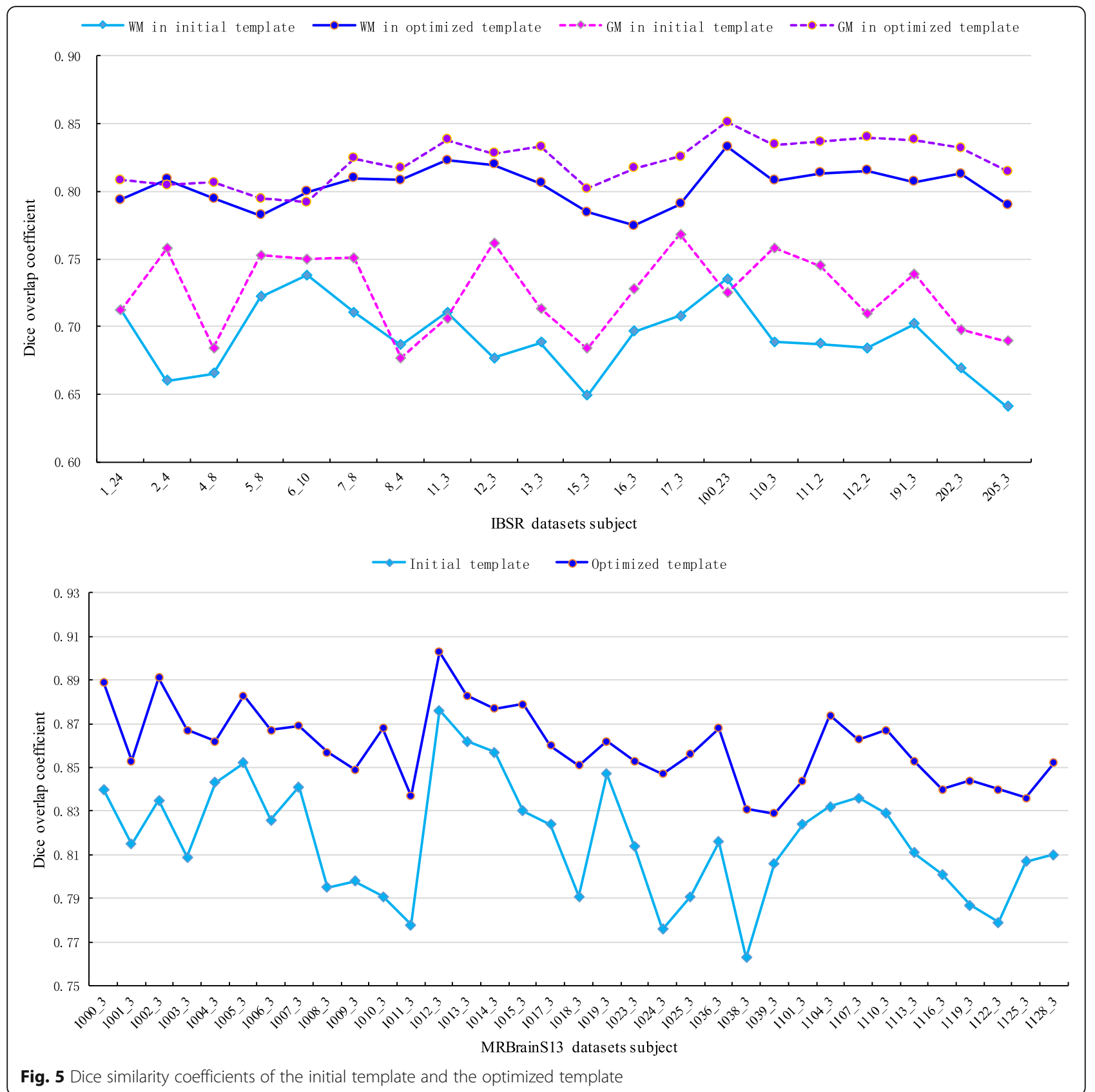

the proposed method in the image segmentation of each subject. In the IBSR datasets, we obtained an average WM Dice coefficient of $0.853 \pm 0.03$ and an average WM Dice coefficient of $0.827 \pm 0.04$. Subject 100_23 had the best segmentation performance, with a WM Dice coefficient of 0.897 and a WM Dice coefficient of 0.873 , while subject 58 had the lowest GM Dice coefficient of 0.806 , and subject 15 3 had the lowest WM Dice coefficient of 0.773 . In the thalamic segmentation of the MRBrainS13 dataset, we obtained an average Dice coefficient of $0.927 \pm 0.014$ and an average HD of $2.92 \pm 0.53$. Subject 1019_3 had the best segmentation performance, with a Dice coefficient of 0.957 and an HD of 1.414. Subject 1002 3 had the worst segmentation performance, with a Dice coefficient of 0.896 and an HD of 4.472 .

A sample of our segmentation results is shown in Fig. 6. It can be observed that our method obtained satisfactory WM, GM and thalamic segmentation performances, and the target organization boundary is smooth, which is beneficial to the three-dimensional reconstruction of the target organization. 
Table 2 Performance index of our method for IBSR datasets and MRBrainS13 datasets. $D_{G M}, D_{W M}$ and $D_{\text {Th }}$ represent the average dice similarity coefficient of GM, WM and thalamics. $D_{\text {Th }}$ represents the average HD of thalamics

\begin{tabular}{|c|c|c|c|c|c|c|c|c|c|c|c|}
\hline \multicolumn{4}{|c|}{ IBSR datasets: } & \multicolumn{2}{|c|}{ Average $D_{G M}$} & 0.853 & \multicolumn{3}{|c|}{ Average $D_{W M}$} & \multicolumn{2}{|l|}{0.827} \\
\hline subject & $D_{G M}$ & $D_{W M}$ & subject & $D_{G M}$ & $D_{W M}$ & subject & $D_{G M}$ & $D_{w M}$ & subject & $D_{G M}$ & $D_{W M}$ \\
\hline 1_24 & 0.822 & 0.803 & $2 \_4$ & 0.829 & 0.790 & $4 \_8$ & 0.809 & 0.803 & 5_8 & 0.806 & 0.797 \\
\hline 6_10 & 0.812 & 0.793 & 7_8 & 0.832 & 0.839 & 8_4 & 0.834 & 0.817 & $11 \_3$ & 0.889 & 0.861 \\
\hline $12 \_3$ & 0.823 & 0.855 & $13 \_3$ & 0.877 & 0.860 & $15 \_3$ & 0.819 & 0.773 & $16 \_3$ & 0.869 & 0.814 \\
\hline 17_3 & 0.893 & 0.822 & 100_23 & 0.897 & 0.873 & 110_3 & 0.883 & 0.810 & $111 \_2$ & 0.875 & 0.854 \\
\hline $112 \_2$ & 0.878 & 0.865 & 191_3 & 0.866 & 0.848 & 202_3 & 0.887 & 0.833 & 205_3 & 0.855 & 0.836 \\
\hline \multicolumn{4}{|c|}{ MRBrainS13 datasets: } & \multicolumn{2}{|c|}{ Average $D_{T h}$} & 0.927 & \multicolumn{3}{|c|}{ Average $\mathrm{H}_{\mathrm{Th}}$} & \multicolumn{2}{|l|}{2.923} \\
\hline subject & $\mathrm{D}_{\text {Th }}$ & $\mathrm{H}_{\text {Th }}$ & subject & $\mathrm{D}_{\text {Th }}$ & $\mathrm{H}_{\text {Th }}$ & subject & $\mathrm{D}_{\text {Th }}$ & $\mathrm{H}_{\text {Th }}$ & subject & $\mathrm{D}_{\text {Th }}$ & $\mathrm{H}_{\text {Th }}$ \\
\hline $1000 \_3$ & 0.928 & 2.828 & 1001_3 & 0.896 & 4.472 & 1002_3 & 0.914 & 3.162 & 1003_3 & 0.934 & 2.236 \\
\hline 1004_3 & 0.92 & 2.828 & 1005_3 & 0.921 & 3.162 & 1006_3 & 0.92 & 3.606 & 1007_3 & 0.938 & 2.236 \\
\hline 1008_3 & 0.942 & 2.828 & 1009_3 & 0.928 & 2.828 & 1010_3 & 0.916 & 3.162 & 1011_3 & 0.924 & 2.828 \\
\hline 1012_3 & 0.903 & 4.123 & 1013_3 & 0.924 & 3.162 & 1014_3 & 0.941 & 2.236 & 1015_3 & 0.937 & 2.828 \\
\hline 1017_3 & 0.91 & 3.606 & 1018_3 & 0.937 & 2.828 & 1019_3 & 0.957 & 1.414 & 1023_3 & 0.917 & 3.606 \\
\hline $1024 \_3$ & 0.93 & 2.828 & 1025_3 & 0.931 & 2.282 & 1036_3 & 0.94 & 2.236 & 1038_3 & 0.931 & 2.828 \\
\hline 1039_3 & 0.906 & 3.606 & 1101_3 & 0.943 & 2.236 & 1104_3 & 0.936 & 2.828 & 1107_3 & 0.948 & 2.236 \\
\hline 1110_3 & 0.932 & 2.828 & 1113_3 & 0.937 & 2.828 & 1116_3 & 0.918 & 3.606 & 1119_3 & 0.915 & 3.162 \\
\hline 1122_3 & 0.921 & 3.162 & 1125_3 & 0.907 & 2.828 & 1128_3 & 0.938 & 2.828 & & & \\
\hline
\end{tabular}

Figure 7 shows the means and standard deviations of the Dice, Recall, Precision and HD coefficients in the MRBrainS13 datasets. It can be observed from Fig. 7a and Fig. $7 \mathrm{~b}$ that the proposed method results in a substantially higher average similarity coefficient and a smaller HD than WV [13], SIMPLE [14], MUSE [15] and MALSF [16]. The target organization segmented by our method is closer to manual expert segmentation than the methods mentioned above. As shown in Fig. 7c and Fig. 7d, the proposed method obtains the best Recall coefficient and the second-best Precision coefficient. This means that the proposed method results in the fewest unrecognized target tissue pixel points, while MALSF results in the fewest misidentified target tissue pixel points.

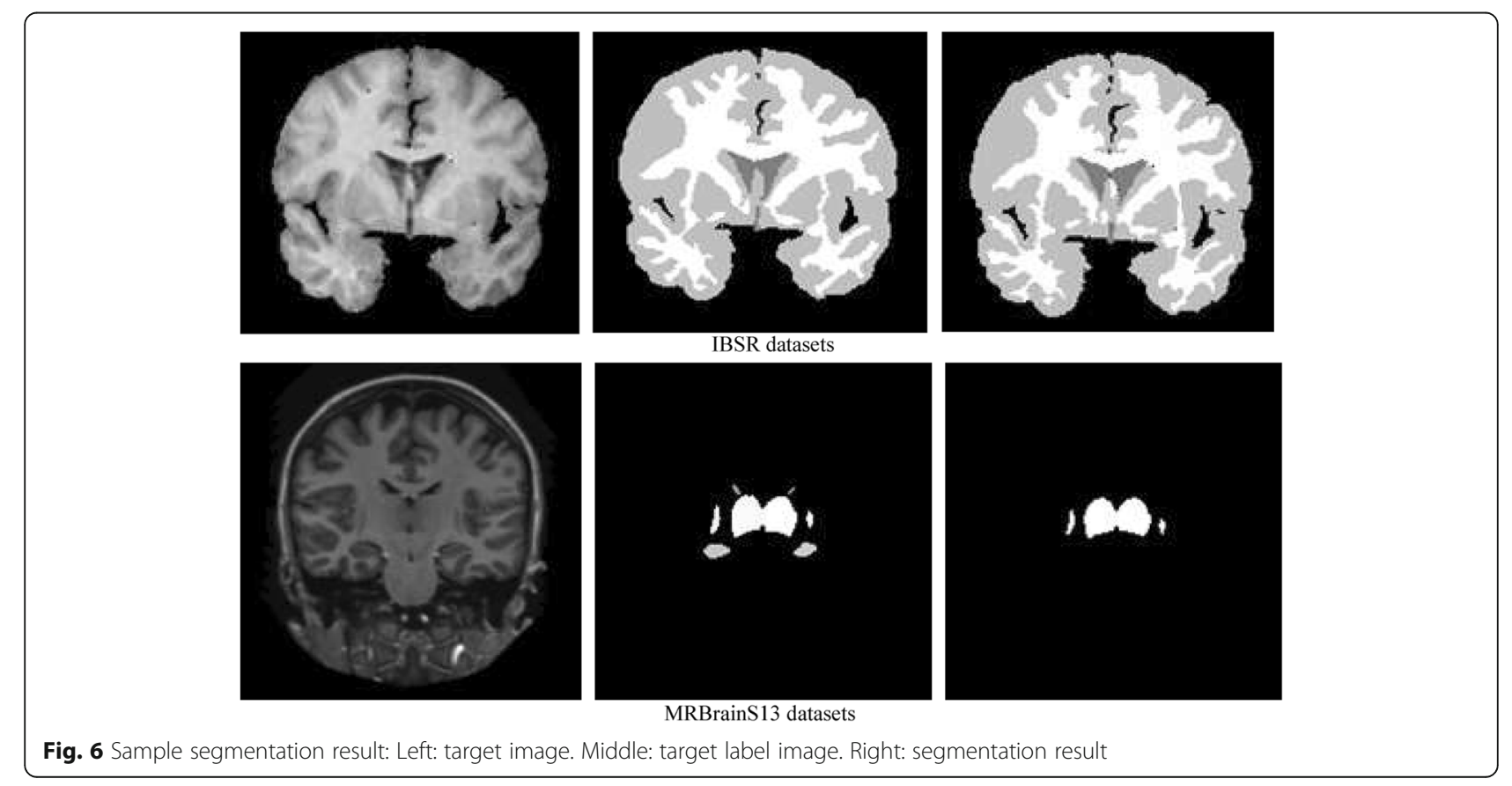



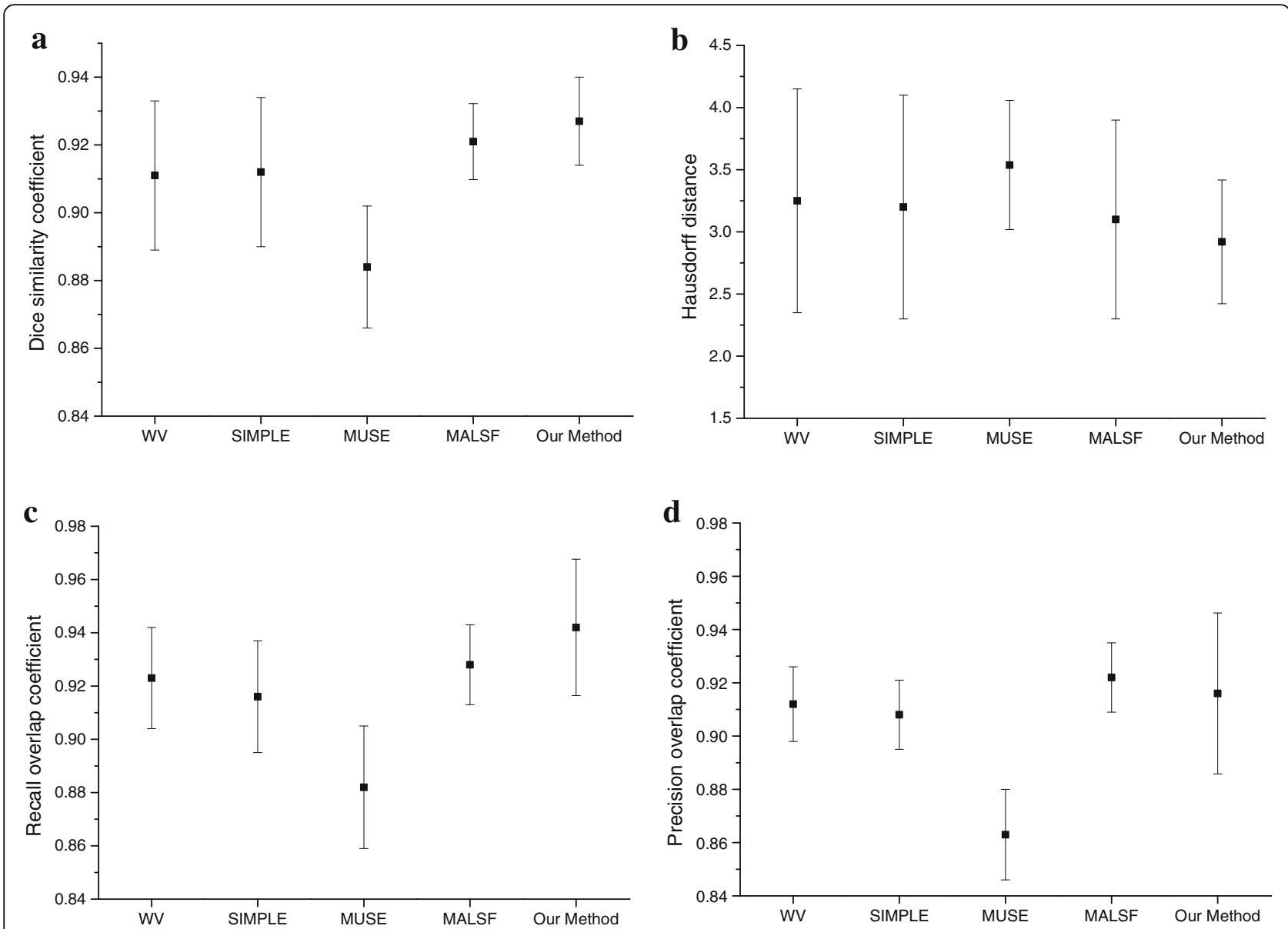

Fig. 7 Performance indexes for the thalamic segmentation of the MRBrainS13 datasets, (a) Dice similarity coefficient, (b) Hausdorff distance, (c) Recall overlap coefficient, (d) Precision overlap coefficient

Currently, our method is implemented with Visual Studio 2012 on an Intel(R) Core(TM) $3.70 \mathrm{GHz}$ CPU, and the average time for segmenting a new image was approximately $2 \mathrm{~min}$.

\section{Discussion}

Usually, MR image analysis is performed by a doctor through the identification and marking of various tissues in the image. However, this work requires a greater professional level of doctor and has high subjectivity. Automatic and semi-automatic image segmentation methods can effectively remove human subjective influences and can effectively reduce segmentation time and improve segmentation accuracy. This kind of method has become a research hotspot in the field of image segmentation.

At present, many automatic and semi-automatic segmentation methods have been proposed. Valverde et al. [28] tested several commonly used automatic image segmentation methods on IBSR datasets, such as fuzzy c-means (FCM) [29] and SPM8 [30]. Tohka et al. [31] proposed a novel MR image segmentation method (SVPASEG) based on a local Markov random field (MRF) and obtained satisfactory brain MR image segmentation results. Mahmood et al. [32] proposed an automatic MR image segmentation method combining mean shift, a priori spatial tissue probability maps and FCM (PPM- FCM). Bendib et al. [33] extended the stationary wavelet transform feature extraction method and used the extracted features to feed a random forest classifier (SWT- RF). They trained and tested this classifier on the IBSR datasets and obtained better WM and GM segmentation results.

Figure 8 shows the performance of our method and the above methods on the IBSR datasets. In WM segmentation, the proposed method obtained the second highest Dice coefficient, similar to that of the BS-CNN method. In GM segmentation, the BC-CNN method obtained the best segmentation performance, and we obtained the third highest Dice coefficient. This is mainly due to the errors generated in the WM segmentation process affecting the performance of the GM segmentation. To verify the effect of WM segmentation errors on GM segmentation, we used subject 205_3 as the target 


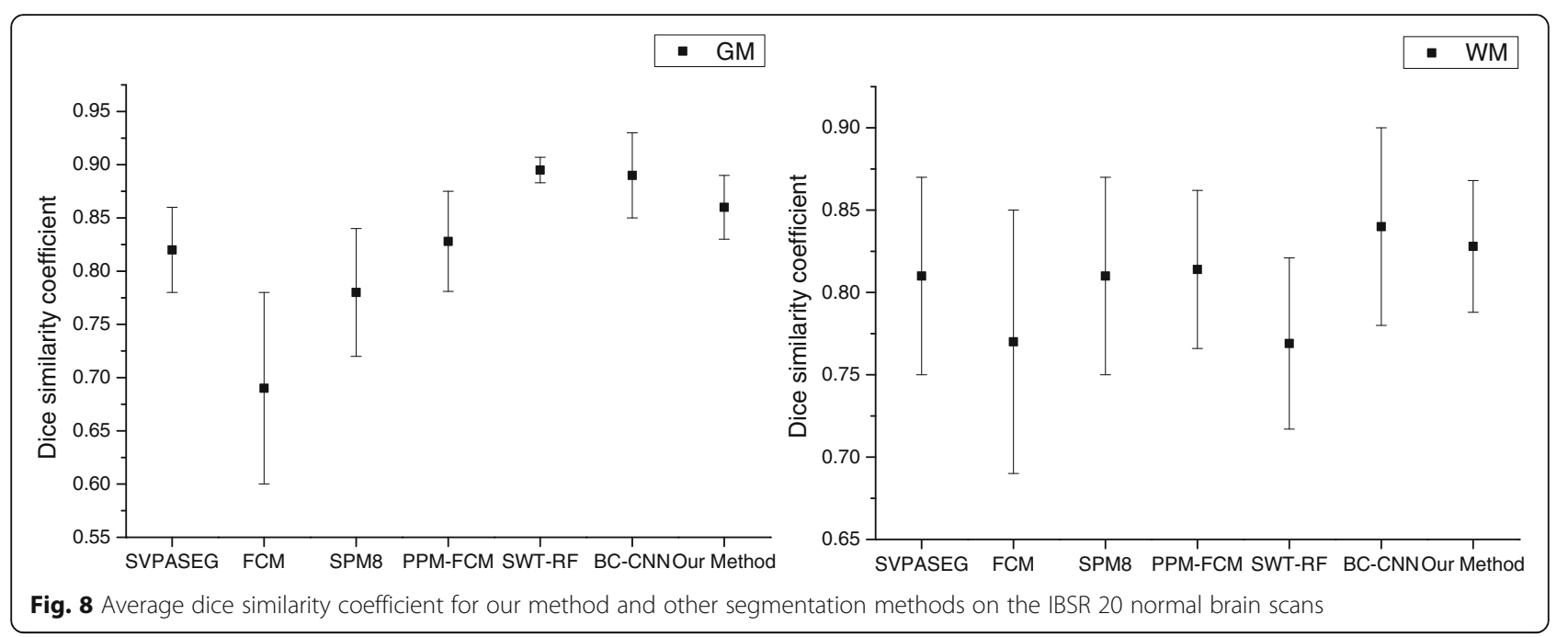

dataset and introduced the WM segmentation errors into the target label image. We obtained an average GM Dice coefficient of 0.887 between the original target label image and the images with errors. This shows that the best Dice similarity coefficient for GM segmentation is 0.887 due to the effect of WM segmentation errors, and we obtained a GM segmentation result of 0.855 .

Multi-atlas registration results have been shown to have a greater impact on segmentation accuracy [16]. The registration result is mainly affected by two factors. One is the choice of registration method, and the other is the selection of the atlas datasets. The more similar the target image and the images of the atlas dataset are, the better the multi-atlas registration results that will be obtained. Selecting a high-precision nonlinear registration method can achieve better registration results, but these methods are expensive to calculate. In the multi-atlas registration, nearly one thousand image registration steps would be carried out, and it would thus take a long time to use the highly complex nonlinear registration method. To reduce the multi-atlas registration time, we chose an image registration method based on affine transformation and then used the template optimization algorithm to improve the registration-induced errors. In our test, the multi-atlas registration process for each target image in the IBSR datasets took approximately $255 \mathrm{~s}$, and in the MRBrainS13 datasets took approximately $409 \mathrm{~s}$.

In the IBSR datasets WM segmentation, we obtained an average Recall coefficient of $0.836 \pm 0.028$ and an average Precision coefficient of $0.823 \pm 0.034$. In the IBSR datasets GM segmentation, we obtained an average Recall coefficient of $0.860 \pm 0.037$ and an average Precision coefficient of $0.849 \pm 0.018$. The Recall coefficients are higher than the Precision coefficients in the segmentation of the two datasets, which means that the pixel points misidentified as the target tissue in the segmentation result are relatively large. In the next study, we will analyse the reasons for this result.

Although the proposed method has been successfully applied to both IBSR datasets and MRBRainS13 datasets, there are still some limitations that affect segmentation accuracy and speed, and these limitations can be further improved. The proposed method can also be used for segmentation of different tissues of other datasets. In our next work, we will improve the limitations of the proposed method and apply it to the segmentation of different organizations of more datasets.

\section{Limitations}

1) The initial template quality has a great impact on the segmentation results and is affected by the selection of atlas datasets and registration methods.

2) In our experiments, the initial search area was set according to the organization outline of the initial template during the template optimization process. If there is a large difference between the images, the template optimization method may not find the real boundary. For example, the location and shape of the diseased tissue have a large randomness, which results in a large difference between the images.

3) The multi-atlas registration process takes a long time, but not all image registrations are meaningful, such as registration between two very different images.

4) The proposed method cannot adapt to the segmentation of small-thickness tissues (such as cortex), because the target profile with small thickness will cause ACM to fall into local extremes. 


\section{Future directions}

1) Combine different atlas datasets and different registration methods to improve initial template quality.

2) Expand the initial search area and redefine the appropriate weight function to expand the capture range of the tissue contour points.

3) Select appropriate images in the atlas datasets for multi-atlas registration will not only save much time but also not reduce the accuracy of the segmentation. How to go about choosing the appropriate images will be a challenging exercise.

\section{Conclusion}

In this paper, we propose a semi-automatic brain image segmentation method. First, a multi-atlas registration method is used to obtain the prior shape information of the target tissue, and then a label fusion algorithm is used to generate the initial template. Second, a template optimization algorithm is used to reduce the multi-atlas registration errors and generate a IAC. Finally, a ACM is used to segment the target tissue. Our method combines the advantages of multi-atlas registration and ACM. The IAC of the target tissue is obtained by multi-atlas registration and template optimization, and it can effectively reduce the influence of human subjective factors compared to manually setting the IAC. Then, a ACM is used to obtain a smooth, continuous target contour. (Fig. 4).

To verify the performance of our method in brain segmentation, we validated our method on two datasets, the IBSR and MRBrainS13 datasets. Experimental results demonstrate the following:

1) Compared with [16], we use the multi-atlas registration method based on affine transformation and use template optimization to improve the registration error. Our method effectively reduces the multi-atlas registration time and achieves higher segmentation accuracy.

2) Compared with the currently used automatic segmentation method, our method obtains better segmentation performance.

\footnotetext{
Abbreviations

ACM: Active contour model; BC-CNN: 3D Convolutional Neural Network Segmentation Method Combined with Boundary Correction; CNNs: Convolutional neural networks; CSF: Cerebrospinal fluid; FCM: Fuzzy cmeans; FCNs: Fully convolutional networks; GM: Gray matter; HD: Hausdorff distance; IAC: Initial active contour; MALSF: Multi-atlases level set framework; MAS: Multi-atlas segmentation; MRF: Markov random field; MUSE: Multi-atlas region segmentation utilizing ensembles; NCC: Normalized correlation coefficient; PPM-FCM: An automatic MR image segmentation method combining mean shift, priori spatial tissue probability maps and FCM; ROI: Regions of interest; SIMPLE: A selective and iterative method for performance level estimation; SVM: Support vector machine; SVPASEG: A MR image segmentation method; SWT-RF: A segmentation method combining stationary wavelet transform and random forest; WM: White matter; W: Weighting voting
}

\section{Acknowledgements}

The author would like to thank the MRbrains13 website and the Center for Morphometric Analysis Internet Brain Segmentation Repository for providing image data.

\section{Funding}

This research was supported by the program for National Natural Science Foundation of China (No.61572159), NCET (NCET-13-0756) and Distinguished Young Scientists Funds of Heilongjang Province (JC201302). The funding body didn't have any influence on the collection, analysis, and interpretation of data or on writing the manuscript.

\section{Availability of data and materials}

The datasets used and/or analysed during the current study are available from the corresponding author on reasonable request.

\section{Authors' contributions}

MW conceived the research. PL performed the experiments and analyses. PL and FL wrote and reviewed the manuscript. All authors read and approved the final manuscript.

Ethics approval and consent to participate

Not applicable.

Consent for publication

Not applicable.

Competing interests

The authors declare that they have no competing interests.

\section{Publisher's Note}

Springer Nature remains neutral with regard to jurisdictional claims in published maps and institutional affiliations.

Received: 25 December 2018 Accepted: 14 May 2019

Published online: 24 May 2019

\section{References}

1. Fox NC, Schott JM. Imaging cerebral atrophy: normal ageing to alzheimer's disease. Lancet. 2004;363:392-4.

2. Durelli $L$, et al. Every-other-day interferon beta-1b versus once-weekly interferon beta-1a for multiple sclerosis: results of a 2-year prospective randomised multicentre study (INCOMIN). Lancet. 2002;359:1453-60.

3. Theodore WH, Fisher RS. Brain stimulation for epilepsy. Lancet Neurol. 2004; 3:111-8.

4. Kass M. Snakes : active Countour models. Int J Comput Vis. 1988;1:321-31.

5. Gorthi S, et al. Active deformation fields: dense deformation field estimation for atlas -based segmentation using the active contour framework. Med Image Anal. 2011;15:787-800.

6. Chakraborty A, Staib LH, Duncan JS. Deformable boundary finding in medical images by integrating gradient and region information. IEEE Trans Med Imaging. 1996;15:859-70.

7. Yeo SY, et al. Segmentation of biomedical images using active contour model with robust image feature and shape prior. Int J Numer Method Biomed Eng. 2014;30:232-48.

8. Heckemann RA, et al. Automatic anatomical brain mri segmentation combining label propagation and decision fusion. Neuroimage. 2006;33: $115-26$.

9. Wang M, Li P. A review of deformation models in medical image registration. J Med Biol Eng. 2019;39:1-17.

10. Iglesias JE, Sabuncu MR. Multi-atlas segmentation of biomedical images: a survey. Med Image Anal. 2015;24:205-19.

11. Aljabar $P$, et al. Multi-atlas based segmentation of brain images: atlas selection and its effect on accuracy. Neuroimage. 2009;46:726-38.

12. Wu G, et al. Hierarchical multi-atlas label fusion with multi-scale feature representation and label-specific patch partition. Neuroimage. 2015;106: 34-46.

13. Artaechevarria $X$, Munozbarrutia A, Ortizdesolorzano C. Combination strategies in multi-atlas image segmentation: application to brain mr data. IEEE Trans Med Imaging. 2009;28:1266-77. 
14. Langerak TR, et al. Label fusion in atlas-based segmentation using a selective and iterative method for performance level estimation (SIMPLE). IEEE Trans Med Imaging. 2010;29(12):2000-8.

15. Doshi J, et al. Muse: multi-atlas region segmentation utilizing ensembles of registration algorithms and parameters, and locally optimal atlas selection. Neuroimage. 2016;127:186-95.

16. Zhang $\mathrm{M}$, et al. Automatic thalamus segmentation from magnetic resonance images using multiple atlases level set framework (malsf). Sci Rep. 2017;7(1)

17. Bai $W$, et al. Multi-atlas segmentation with augmented features for cardiac mr images. Med Image Anal. 2015;19(1):98-109.

18. Zikic D, Glocker B, Criminisi A. Encoding atlases by randomized classification forests for efficient multi-atlas label propagation. Med Image Anal. 2014; 18(8):1262-73.

19. Krizhevsky A, et al. Imagenet classification with deep convolutional neural networks. Commun ACM. 2017;60(6):84-90.

20. Litjens $\mathrm{G}$, et al. A survey on deep learning in medical image analysis. Med Image Anal. 2017;42:60-80.

21. Moeskops $P$, et al. Automatic segmentation of MR brain images with a convolutional neural network. IEEE Trans Med Imaging. 2016;35(5):1252-61.

22. Nie D, et al. Fully convolutional networks for multi-modality isointense infant brain image segmentation. Biomedical imaging (ISBI), 2016 IEEE 13th international symposium on IEEE. 2016;1342-1345.

23. Beibei $\mathrm{H}$, et al. Robust 3D convolutional neural network with boundary correction for accurate brain tissue segmentation. IEEE ACCESS. 2018;6: 75471-81.

24. Sjöberg C, Ahnesjö A. Multi-atlas based segmentation using probabilistic label fusion with adaptive weighting of image similarity measures. Comput Methods Programs Biomed. 2013;110:308-19.

25. Roberts LG. Machine perception of three-dimensional solids. In: Optical and electro-optical information processing. Cambridge, MA: MIT Press; 1965. p. 159-97.

26. Jr YA, et al. A geometric snake model for segmentation of medical imagery IEEE Trans Med Imaging. 1997;16:199-209.

27. Center for Morphometric Analysis Internet Brain Segmentation Repository (IBSR). 2012. https://www.nitrc.org/projects/ibsr.

28. Valverde $\mathrm{S}$, et al. Comparison of 10 brain tissue segmentation methods using revisited ibsr annotations. J Magn Reson Imaging. 2015;41(1):93-101.

29. Pham DL. Spatial models for fuzzy clustering. Comput Vis Image Underst. 2001;84:285-97.

30. Ashburner J, et al. SPM8 Manual. Wellcome Trust Centre for Neuroimaging Institute of Neurology, UCL. 2011.

31. Tohka J, et al. Brain mri tissue classification based on local markov random fields. Magn Reson Imaging. 2010;28(4):557-73.

32. Mahmood Q, Chodorowski A, Persson M. Automated MRI brain tissue segmentation based on mean shift and fuzzy c-means using a priori tissue probability maps. Elsevier Sci Inc. 2015;36(30):185-96.

33. Bendib MM, Merouani HF, Diaba F. Automatic segmentation of brain mr through stationary wavelet transform and random forests. Pattern Anal Applic. 2015;18:829-43.

Ready to submit your research? Choose BMC and benefit from:

- fast, convenient online submission

- thorough peer review by experienced researchers in your field

- rapid publication on acceptance

- support for research data, including large and complex data types

- gold Open Access which fosters wider collaboration and increased citations

- maximum visibility for your research: over $100 \mathrm{M}$ website views per year

At $\mathrm{BMC}$, research is always in progress.

Learn more biomedcentral.com/submissions 